\title{
Canadian Wood Pallets: Is it a Good Deal, EH? AN UNDERGRADUATE CASE IN COST ACCOUNTING
}

\author{
Patrick O’Meara \\ BRC Journal of Advances in Education 4, no. 1 (2020): 97-104. \\ https://dx.doi.org/10.15239/j.brcadvje.2020.04.01.ja07 \\ WEB APPENDIX
}

https://dx.doi.org/10.15239/j.brcadvje.2020.04.01.wa07 
Case Information Tables

Table One

\begin{tabular}{|c|c|c|c|c|c|c|c|c|}
\hline Model & Type & Size & Capacity & Forklift & Weight & \multicolumn{3}{|c|}{ Minimum Pricing } \\
\hline & & & & & & 5 units & 10 units & 20 units \\
\hline $\begin{array}{l}\text { CWP- } \\
1070\end{array}$ & New Wood & $48 " x 40$ & 2,500 & 4-way & 42 & $\$ 29 \mathrm{pu}$ & $\$ 28 \mathrm{pu}$ & $\$ 26 \mathrm{pu}$ \\
\hline $\begin{array}{l}\text { CWP- } \\
850\end{array}$ & Recycled & $48 " \times 40$ & 2,500 & 4-way & 40 & $\$ 20 \mathrm{pu}$ & $\$ 19 \mathrm{pu}$ & $\$ 17 \mathrm{pu}$ \\
\hline
\end{tabular}

Table Two

\begin{tabular}{|l|l|l|}
\hline \multicolumn{3}{|c|}{ Direct Materials Costs } \\
\hline & New Wood Materials & Recycled Wood Materials \\
\hline Top Boards & $\$ 1.50$ per board & $\$ 1.30$ \\
\hline Bottom Board & $\$ 1.50$ & $\$ 1.30$ \\
\hline Support Blocks & $\$ .50$ per block & $\$ .25$ per block \\
\hline
\end{tabular}

Table Three

\begin{tabular}{|l|l|}
\hline \multicolumn{2}{|c|}{ Divisional Budgeted Overhead } \\
\hline Cutting & $\$ 300,000$ \\
\hline Assembly & $\$ 500,000$ \\
\hline Wood Treating & $\$ 150,000$ \\
\hline Total Manufacturing Overhead & $\mathbf{\$ 9 5 0 , 0 0 0}$ \\
\hline
\end{tabular}

Table Four

\begin{tabular}{|l|c|c|c|c|}
\hline \multicolumn{4}{|c|}{ Service Department and Operating Department Data } \\
\hline & $\begin{array}{l}\text { Administration } \\
\text { (employees) }\end{array}$ & & $\begin{array}{l}\text { Infor. Systems } \\
\text { (system hours) }\end{array}$ & \\
\hline Budgeted Overhead & $\mathbf{\$ 2 0 0 , 0 0 0}$ & & $\mathbf{\$ 1 5 0 , 0 0 0}$ & \\
\hline Administration & & $0 \%$ & 200 & $33 \%$ \\
\hline Information Systems & 10 & 20 & & 0 \\
\hline Cutting & 10 & 20 & 100 & 17 \\
\hline Assembly & 20 & 40 & 200 & 33 \\
\hline Wood Treating & 10 & 20 & 100 & 17 \\
\hline Total & $\mathbf{5 0}$ & $\mathbf{1 0 0 \%}$ & $\mathbf{6 0 0}$ & $\mathbf{1 0 0 \%}$ \\
\hline
\end{tabular}


Table Five

\begin{tabular}{|l|l|l|l|}
\hline \multicolumn{4}{|c|}{ Direct Labour Costs by Division } \\
\hline & New Wood & Recycled & Total \\
\hline Cutting & $\$ 200,000$ & $\$ 120,000$ & $\$ 320,000$ \\
\hline Assembly & 250,000 & 225,000 & 475,000 \\
\hline Wood Treatment & 100,000 & 50,000 & 150,000 \\
\hline Total & $\mathbf{\$ 5 5 0 , 0 0 0}$ & $\mathbf{\$ 3 9 5 , 0 0 0}$ & $\mathbf{\$ 9 4 5 , 0 0 0}$ \\
\hline
\end{tabular}

Table Six

\begin{tabular}{|l|l|l|l|l|}
\hline Activity & New Wood & & Recycled Wood & \\
\hline Cutting & 3,000 & MH & 2,000 & MH \\
\hline Assembly & 20,000 & DLH & 17,000 & DLH \\
\hline Wood Treating & 450,000 & Treatments & & \\
\hline
\end{tabular}


Case Figures

Figure One

\section{Block Design}

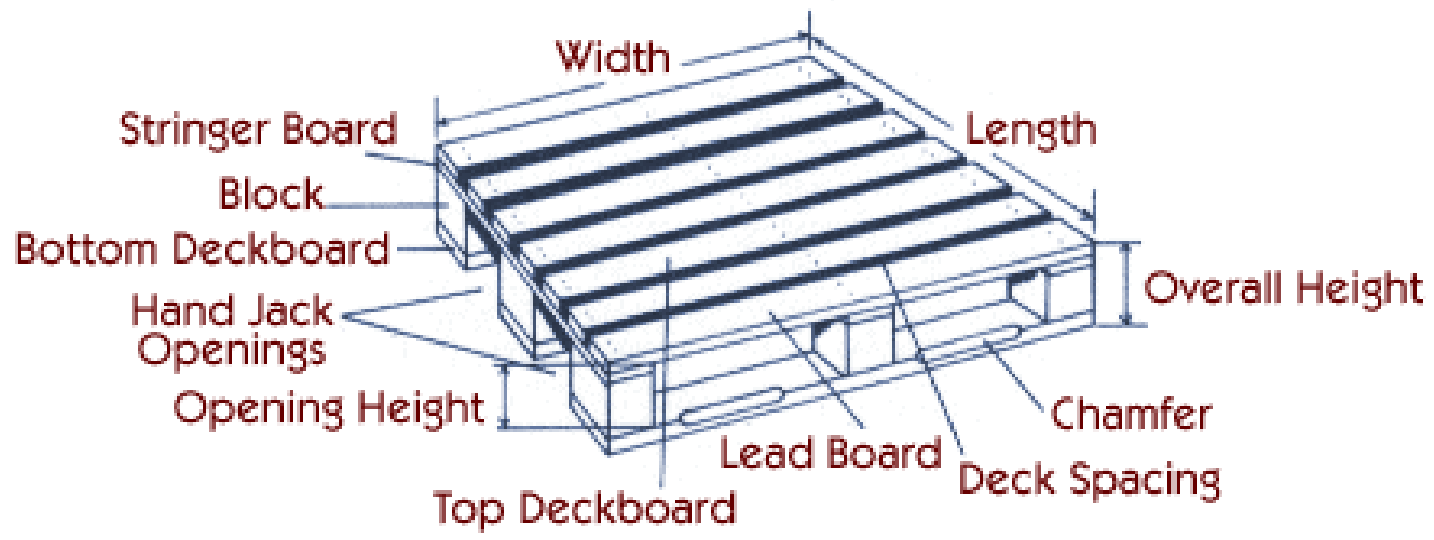

Source: https://associated-pallets.co.uk/what-we-do/pallets-explained/ii

i http://www.canadianpallets.com/ (February, 2019)

ii https://associated-pallets.co.uk/what-we-do/pallets-explained/ (February, 2019) 